\title{
Dynamic Traffic Control and Management System for Smart Cities
}

\author{
Abdul Mateen ${ }^{1 *}$, Sabeen Sher', Amjad Rehman², Zahid Hanif', \\ Tooba Akhtar ${ }^{1}$, Mahmood Ashraf ${ }^{1}$ \\ 1 Department of Computer Science, Federal Urdu University of Arts, Science \& Technology, Islamabad, Pakistan \\ 2 College of Computer and Information Systems, Al Yamamah University, Riyadh,11512, Saudi Arabia \\ * Corresponding author's e-mail: abdulmateen@fuuastisb.edu.pk
}

\begin{abstract}
The computational capabilities of computers enable a human being to control the vehicles and their traffic. Automatic traffic control system not only reduces the effort of human but also provides secure and accurate results. Here, the architecture of Agent-Based Autonomous Controller (ABAC) that manages vehicles at traffic signals intelligently was proposed. The proposed solution is followed by a vehicle counting by infrared (IR) sensor; providing solution for independent and mutual dependent signals for smooth traffic flow; emergency vehicles alert and priority over public vehicles through Radio Frequency Identification (RFID). Finally, the proposed research was tested through simulation that reveals the performance over the previous traffic control and management architectures.
\end{abstract}

Keywords: traffic flow, management, mutual dependent, infrared

\section{INTRODUCTION}

The world's first traffic light, manually operated by gas-lit signal, installed outside the Parliament in London, in December 1868, was short lived. Traffic control management seemed to be necessary in the late 1890s. The first automated traffic control system was installed in 1910 in Chicago. The words "STOP" and "GO" were used instead of signal lights. In 1912, a traffic control device was placed on top of tower at Paris, Rue Montmartre and Grande Boulevard. The first traffic light in South India was installed at Egmore Junction, Chennai in 1953.

Many countries in these days are confronted with great challenges in the road transport sector, as they need to maintain mobility while their main purpose is boosting the economic man power, save time and quality of life. Usually, traffic is managed by three techniques, i.e. manual, automatic and autonomous. In the case of manual one, traffic is controlled by the traffic sergeant; automatic traffic is managed by allocating pre-constant time to each side of the signal, while in the case of autonomous, traffic is managed by observing the traffic on each side of the signal and set time accordingly. There are three different direction of the vehicles at the traffic signal which are left, right and straight [1]. The lights at signal changes as: green to yellow, red to yellow, yellow to green or red as shown in Figure 1. However, green light never changes to red light directly and vice versa.

The mechanism that is used for planning and controls traffic signals for vehicles on roads is known as the traffic management system. Traffic

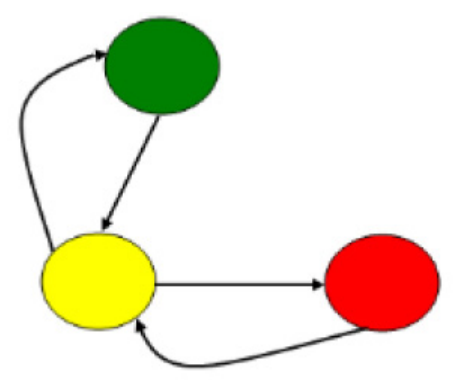

Fig. 1. Traffic signal light sequence 
management is used to control traffic on roads in an effective way. For example, management of vehicles at extra lanes during rush hours and entrance ramp control. The Advanced Traffic Management System (ATMS) is a primary subfield within the Intelligent Transportation System (ITS). The ATMS view is a top-down management perspective that integrates technology primarily to improve the flow of vehicle traffic and safety.

Well-planned arrangements of components such as traffic signals, detectors or sensors and controller play a vital role in any tolling solution. Traffic signal lights are placed beside or above road to guide, warn, and regulate the flow of traffic, which includes motor vehicles, motorcycles, bicycles and pedestrians. An automated Traffic controller system is used to control and coordinate with the traffic lights in order to manage the vehicles at signals. A sensor is used to check the volume of traffic on signal and detection of some specialized vehicles such as ambulance, rescue etc. Nowadays, multiple sensors are used to detect vehicles, e.g. IR sensors, piezoelectric sensors, magnetic sensor and video vehicle detection etc.

IBM introduced the concept of autonomic computing in 2001 [2-6]. A number of systems have used the agent-oriented and autonomic computing approach since then. In agent-oriented systems, there are various agents which work together in order to provide solution of the given problem [7]. In this piece of work, an agent-based architecture is proposed that is autonomous with respect to gathering data from the environment using sensors and making decisions that should handle the traffic loads smoothly in order to mitigate congestions. The solution-based case study was also performed by taking the traffic data of Islamabad, capital of Pakistan. In this case study, the traffic signals were divided into two types, according to their relationship, i.e. independent and dependent signals. Two signals which are connected and close with each other are said to be mutual dependent traffic signals.

The rest of the paper is organized as follows: Section II discusses the previous research and studies that are dedicated to control and manage traffic. Section III presents the architecture of our proposed architecture to remove traffic congestion and handling of rescue vehicle. Section IV discusses the assign time algorithm of mutual dependent signal. Section $\mathrm{V}$ provides case study of mutual dependent signal. Section VI simulates the purposed architecture and its working. It re- flects the effectiveness of the ABAC architecture and finally section VII provides the conclusion and future directions.

\section{RELATED WORK}

There are various studies which were carried out in past for traffic monitoring, control and management. Some of these are discussed as under:

An agent-based traffic system was proposed where expertise of the agents is calculated by comparison of nearby agents and weights are assigned to each [8-9]. The proposed method was tested on the traffic lights and achieved good results.

The traffic management architecture was proposed to handle the emergency vehicle [11-13] through Radio Frequency Identification (RFID). Specific vehicle is identified with coordinates by using RFID and Global Positioning System (GPS). Driver uses buzzer for emergency vehicle to open the signal.

A Digital Traffic Infrastructure Network (DTIN) was introduced [14] where traffic signals are considered as node. Each node has sensing capabilities that can calculate the flow of traffic. Communication from one node to other is provided by the wired network. Each node is managed by the Node Agent (NA) and every emergency vehicle is managed by the Vehicle Agent (VA). The communication between NA and VA is assumed by a wireless. The Monitoring agent (MA) update the NA about the traffic information these mobile MA's migrate from one node to other node to update the NA about traffic conditions. MA queries the NA and in return NA senses the traffic and replies. Then MA updates the traffic map. After updating traffic map, the MA delivers the traffic map to the DTIN. Whenever a road accident happens, MA updates the traffic map as well. The other mobile agent Path Finding Agent (PFA) has the responsibility to construct partial green corridor with the least crowded path. The VA knows the destination of the emergency vehicle and connects with NA. It releases PFA wirelessly. PFA lands on a Node and then the PFA calculate the path according the information available on the traffic map on a node. The algorithm provides the path that is shortest to reach at destination. However, the system is expensive to implement and complex due to wired and wireless network. Moreover, it is also not possible to give separate VA to each vehicle. 
Inductive loop that works on constant frequency [15] was introduced [15], in which wire ends are connected to the Monitor Unit. It uses a switch which is a few meters away from the traffic intersection. Whenever a vehicle is passed at that point an increment occurred in the vehicle count. When an emergency vehicle is reached, it communicates with Road Side Unit (RSU) and receives a tag for emergency vehicle. Due to the Radio Frequency Identification (RFID) on the emergency vehicle, emergency count is incremented and is sent to the Traffic Control Unit (TCU). If there are two or more emergency vehicles at any side, then the TCU turns on the green signal for that side. No manual effort is needed and priority is given to the emergency vehicle that saves time for the ambulances. The proposed system has the ability to work in all the conditions except the rough weather.

Predictive Road Traffic Management System (PRTMS) was introduced in [16]. PRTMS used a communication system to predict the future traffic congestion. Rerouting the vehicles is also included in PRTMS. Central Control Unit (CCU) is used to predict the future traffic congestion based on Linear Algorithm. A Road Side Unit (RSU) is used to exchange information from each intersection. The RSU was connected with the CCU with Wide Area Network (WAN). Every vehicle should have an On Board Unit (OBU) that is responsible of sending trip information including Point of Attraction (POA) through RSU. CCU predicts the traffic flow on every intersection and provides the trip route with minimum time. The RSU and OBU are connected wirelessly. When OBU is entered into the range of RSU, it is detected dynamically. Each RSU broad cast a packet in every second to each OBU. Each OBU sends a unicast response messages to RSU after 5 seconds. The RSU sends the traffic information to CCU through Ethernet link. Then, , the CCU predicts the traffic every minute and sends the route information to a corresponding RSU. The RSU delivers this message by broadcasting. At the same time the CCU changes the cycle time of the intersection. The proposed system depends upon the communication between CCU, RSU and OBU. The system is very complex and expensive; it is not possible to connect every vehicle with OBU country like in Pakistan.

Multi-agent system with two modes was introduced [17-18]; in the first mode, an agent learns from other agents without cooperation but in the second mode, an agent learns from the other nearest agent through an algorithm. In the proposed system, emergency vehicles were given no priority and there is no explanation how these agents communicate with each other. Moreover, the system is much too slow and cannot work properly in poor weather conditions.

Two modules were proposed in [19] where system transmitter and receiver were used. When an emergency vehicle comes into the range of the radio waves, then the transmitter module of emergency vehicle sends the radio signal on the road side that is received by the Receiver. After processing the data, the green signal is turned on for the emergency vehicle. When an emergency vehicle crosses the intersection, the green light turns to red. The maximum range for the radio signal is 25 meters which is too short and may cause accidents.

The proposed system, as discussed in [20], obtained input from sensors and image processing techniques. The system proceeds on the number of vehicles and the average speed of the vehicles. Then green light works according to the traffic flow. However, the system is not efficient until all the intersection have communication and synchronization with each other. The other thing is that getting the input is major issue and crucial but in this system the mechanism of obtaining inputs is not clear, though the system seems to be very fast.

In the research that was presented in [21-22], the numbers of vehicles at each lane were counted and the Round Robin Scheduling Algorithm was applied. In an Intelligent Traffic Management System (ITMS), vehicles are assumed; otherwise, the number of vehicles is taken from the sensors or by applying image processing or some other technique. In the proposed system, there is no priority for emergency vehicles and ITMS framework may fail if any mishap occurs at the traffic control point.

The proposed work for traffic management is different from the above-mentioned studies in many ways. In this research, an IR sensor was used to detect and count vehicles, which is the most efficient and cheapest way. Unlike previous studies, algorithms for independent as well as dependent signals were explained. A rescue vehicle is detected through RFID and handled intelligently at all the traffic signals without any panic and delay. Another difference is the dynamically allocation of signal cycle time (total time for all four sides). It will provide the architecture 
that constitutes an optimized solution for traffic management by considering all relevant factors and constraints. Each vehicle was given time in such a manner that the average waiting time was reduced. The proposed traffic management architecture was also tested through a simulation.

\section{THE ABAC TRAFFIC MANAGEMENT ARCHITECTURE}

When there is huge traffic, autonomous agents are the better option to handle them. The Agent-Based Autonomous Controllers (ABAC) architecture for traffic management, where each component of the proposed architecture is represented by an autonomous agent, was discussed. Here, these components were not discussed in detail, as they have already been discussed in our previous work.

\section{Environmental Components}

There are several ways to detect and count vehicles on road and traffic signal [10]. One method to detect a vehicle is by using IR Sensors. For each of the four roads that comes to junction have IR obstacle sensor modules that are installed at each lane. The IR sensor is also placed on the exit point of the road. These IR sensor modules only detect the obstacle which crosses the IR Beam. Each IR sensor works together with the Arduino board which is installed on each lane of the road. Each lane of the road is about 8 to 10 feet wide. Therefire, the range of IR Sensors is set to detect any vehicle that comes in the range of 8 to 10 feet only. Each road side has 4 lanes of 10 feet wide and each IR obstacle Sensor Modules Set consists of 4 IR sensors with the Arduino Uno board to detect and count vehicles that pass through these lanes.

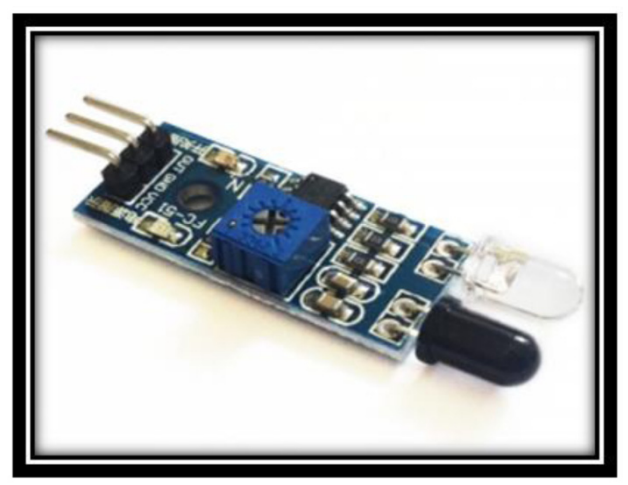

Fig. 2. IR Obstacle Module
Figure 2, shows the IR Obstacle module [2425] which has 3 pins; 2 pins are used for power up the module that is $5 \mathrm{~V} \mathrm{DC}$ and $3 \mathrm{rd}$ pin is used for output. This module is connected to the Arduino Uno Board. The output pin of the module is attached to analog pin 2 of the Board. The IR module is installed at each lane of the road. The working principle of the IR Obstacle module [26] in which IR transmitter throw IR beam on object and it reflects back when any vehicle comes in its way. The beam reflected back is then sensed by the IR Receiver. The detection range can be changed by setting up the variable mounted on the IR Obstacle Module.

The value measured by the Analog pin of Arduino Uno is approximately 1000, when there is no vehicle. This value may decrease up to 200 when vehicle obstacles come into its range. When a vehicle is detected, a pulse (binary 1 ) is sent to the Main PIC controller to add the count by 1 to the vehicle count of that side. Note that for each lane of the road there the number of IR Sensors installed corresponds to the number of lanes. Therefore, if four vehicles come at a time on the road, an IR sensor is installed on each lane to detect only its own lane vehicle and send binary 1 to the main controller. Hence, vehicles are counted by the main controller and the same procedure is applied for the vehicles which exit from the exit point of the road junction. The IR sensor modules installed at the exit point of each lane send a pulse to the main controller when the vehicle obstacle is sensed, which deduces the counting variable value by 1 for each sensor of each lane of the road. This procedure is repeated for the signal to count the vehicles at each side of the signal. The road counting is then considered by time allocation algorithm at the end of each timing cycle.

Nowadays, the number of vehicles on roads increases rapidly. These vehicles include cars, motorbikes, cabs, buses, trucks, as well as emergency vehicles like ambulances, fire brigade vehicles, police cars, VIP vehicles etc. Emergency vehicle need to be detected to give them priority over other public vehicles. Normal vehicles are detected through IR sensors, but it is not enough for detecting emergency vehicles.

In order to detect emergency vehicles, Radio Frequency Identification (RFID) technology is used. RFID tags are mounted on the vehicles that are used for emegencies. Each RFID tag has a unique ID number that is registered to a single 
emergency vehicle in a database. Each RFID tag contains important information of the emergency vehicle e.g., Vehicle Registration number, type (Ambulance, VIP, Fire brigade, police etc.,), Engine number, chassis number etc.

The mounted RFID tags are detected by the RFID readers that are located on each lane of each road, approximately 500 meter away from the signal. Each emergency vehicle has their own RFID mounted Tag. When an emergency vehicle comes on the road at 500 meter away from the signal it is detected by RFID readers. As shown in Figure 3, a RFID reader reads the unique tag ID and relates the information from the database. If it is a valid Tag ID, then it gives signal to the DM to turn all signals to red, and green of the road from which an emergency vehicle is coming. Before turning all signals to red, the current state of signals is saved. When an emergency vehicle crosses the signal, DM restore signal to its previous state. For each side of the road, there are two RFID Readers: one installed on the entry and other on the exit point of junction.

Figure 4, shows the wiring diagram of RFID reader to arduino board [28].

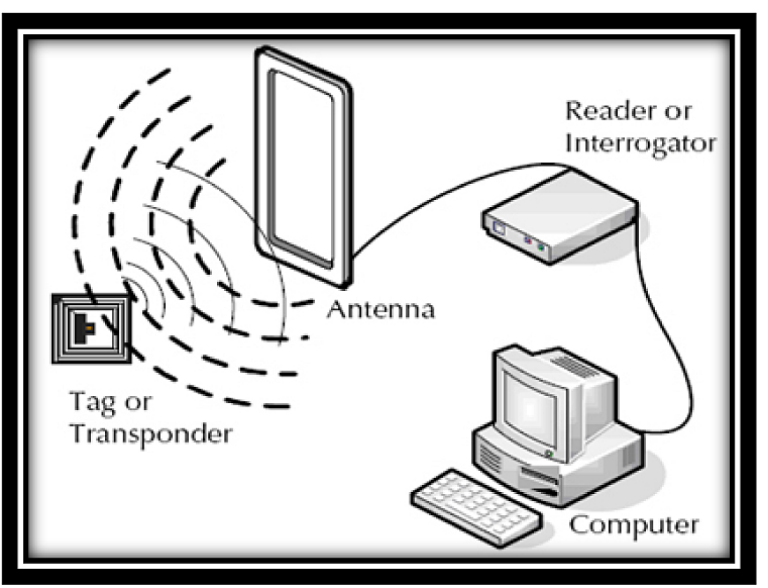

Fig. 3. RFID Working

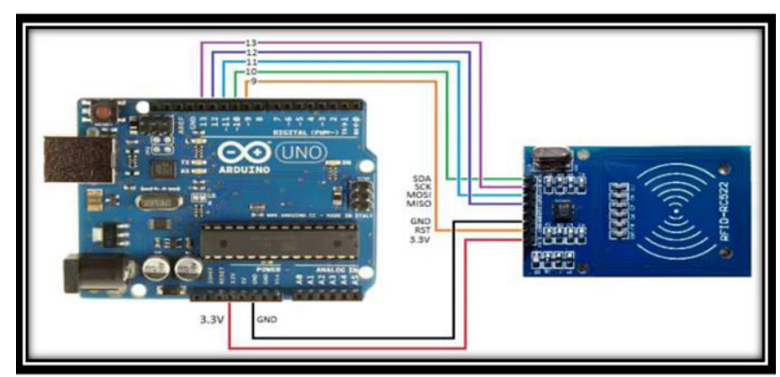

Fig. 4. RFID Reader Wire

\section{MESSAGE FORWARDING FOR EMERGENCY VEHICLE APPROACHES TO NEXT SIGNAL}

When an emergency vehicle is detected by the RFID Readers on the exit point of signal junction, a message is sent to the corresponding next signal about the incoming emergency vehicle. This message contains the information about the detected emergency vehicle, like the Vehicle Registration number, its type, and approximated time to reach the next signal junction.

As shown in Figure 5, it uses 2 HC-12 modules for wireless serial communication instead of physical wired commutation. When one microcontroller sends data to other microcontroller wirelessly it needs two HC-12 modules. Each one is connected to the one microcontroller. On the transmitting side, a microcontroller is connected to module in such a way that the transmitting pin of the controller is connected to the receiving pin of module and the receiving pin of the controller is connected to the transmitting pin of the module. The same connection scheme is repeated on the receiving side for second module and its microcontroller [29].

Whenever a HC-12 module receives data on its receiving pin RXD, it automatically sends a signal wirelessly using radio waves. At the same time, the second $\mathrm{HC}-12$ module receives data wirelessly and sends a signal to microcontroller from its transmitting pin TXD. This module allows half-duplex communication, meaning at a time, one module sends data and the other receives it, and next time the other module sends signal, while the other module receives it. Both modules cannot send or receive data over the air at the same time [29].

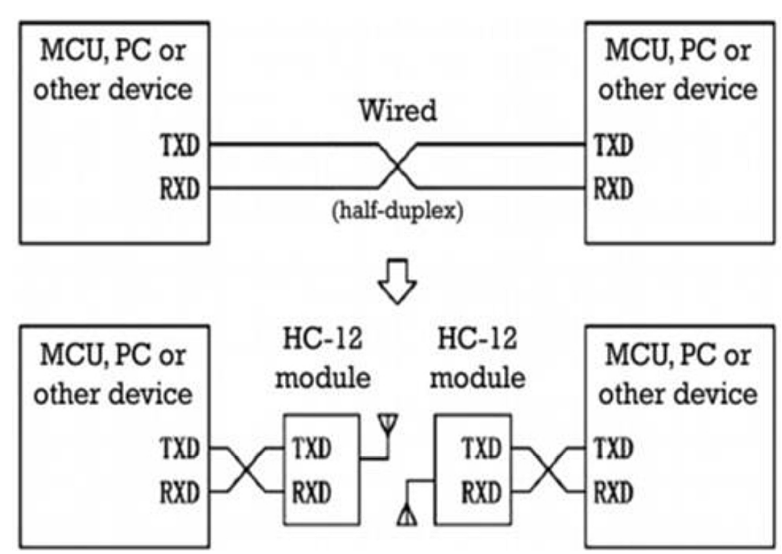

Fig. 5. Working Principle of HC-12 
This communication of message to next signal is done by using HC-12 (Wireless serial port communication) module. HC-12 uses multiple channels (up to 100 channels) within range of 433 to $437 \mathrm{MHz}$, each communication channel is about $400 \mathrm{KHz}$ bandwidth. Maximum $100 \mathrm{~mW}$ of power is consumed in transmission at $20 \mathrm{dBm}$. For longer distance communication, the serial baud rate should be reduced [29].

As multiple channels are available for communication, different channels are used for communication between four different directions of junction but similar channels for corresponding two signals (line of sight). This wireless communication takes place up to 2 kilometer distance using half duplex serial communication link. For reliable communication, the baud rate must be 1200 for longer distances. If we want to communicate over greater distances, we must set repeaters appropriately.

When a signal receives the input information from HC-12 module, the current information is analyzed and the time to approach the signal is calculated, then it is sent to a signal controller which then turns signal to green for an incoming emergency vehicle, before it approaches the signal. After an emergency vehicle passes from the signal, the previous signal cycle is restored and the information on the exit of this emergency vehicle is sent to the next corresponding signal.

\section{Knowledge Base}

A database which stores the traffic intensity, threshold of each signal, traffic flow, distance of next connected signals at each side, emergency alarms and RFID tag information e.g. (ID, Vehicle Registration number, type).

\section{Observer}

It comprises an Analyzer, Decision Making (DM) and Learner agent.

Analyzer Agent: It takes the input from the both IR sensor and RFID reader, makes decision about the dependent as well as mutual independent signal on the basis of distance to next signal and process accordingly. The traffic flow information is sent to DM agent. On the other hand, when a rescue sound is detected then DM agent is called for quick response.

Decision Making Agent: It receives two types of inputs from the Analyzer agent, i.e. vehicle count from the IR sensor and emergency vehicle sound.
In the case of IR sensing, the assigned time is calculated by adding the exit time of first and next rows. The exit time for first row is calculated by using the formula $3600 / 10000 *$ Distance, where $3600 / 10000$ represents the time to cover a meter when the vehicle's average speed is $10 \mathrm{~km} /$ hour. This policy is adopted as vehicle initiates with 0 speed and finally reaches up to $20 \mathrm{~km} /$ hour when exits it the signal. The exit time for the next rows is calculated for 2 meters which is the distance of vehicle from its successor row. All four sides were assigned time by using this strategy. Finally, the signal is opened that has minimum time. Signal cycle time is updated on regular basis. when there is mutual dependent signal, first sum up the number of vehicles of the dependent signal, $70 \%$ from straight signal. After summation, the assigned time is calculated by adopting the same startegy as mentioned for independent signal. However, when a sound is received from rescue vehicle, DM agent stores the current sequence into the knowledge base, restoring the signal sequence after 10 sec. The information about an emergency vehicle is passed to next signal for making arrangement of rescue vehicle passeged without any wait.

Learner Agent: It takes data from the repository, with traffic flow patterns and updates the knowledge base if necessary that is further used by the Analyzer and DM agent in future.

\section{MUTUAL DEPEDENT SIGNAL ASSIGN TIME ALGORITHM}

Mutual Signal Assign Time Algorithm (Figure 6) is used to calculate the assigned time of all four sides of both the signals S1 and S2 as shown in (Figure 7) for handling huge traffic in an effective way. This algorithm is invoked on the regular basis in order to update assign time of all sides of the signal and keep it updated according to the traffic flow. Mutual dependent sides of the signal update their assigned time according to the vehicle of the signal on which they are dependent.

Total Signal Time (TST) of both Mutual Dependent signal should be the same. In the case when the TST of both signals is not same, then the remaining time should be added to the independent sides of the mutual signal with less time, because the signal cycle time will always be the same for both signal S1 and S2. E.g. In first row of Table 2, signal sequence of S1 and S2 are A,C,D,B and E,G,H,F. 


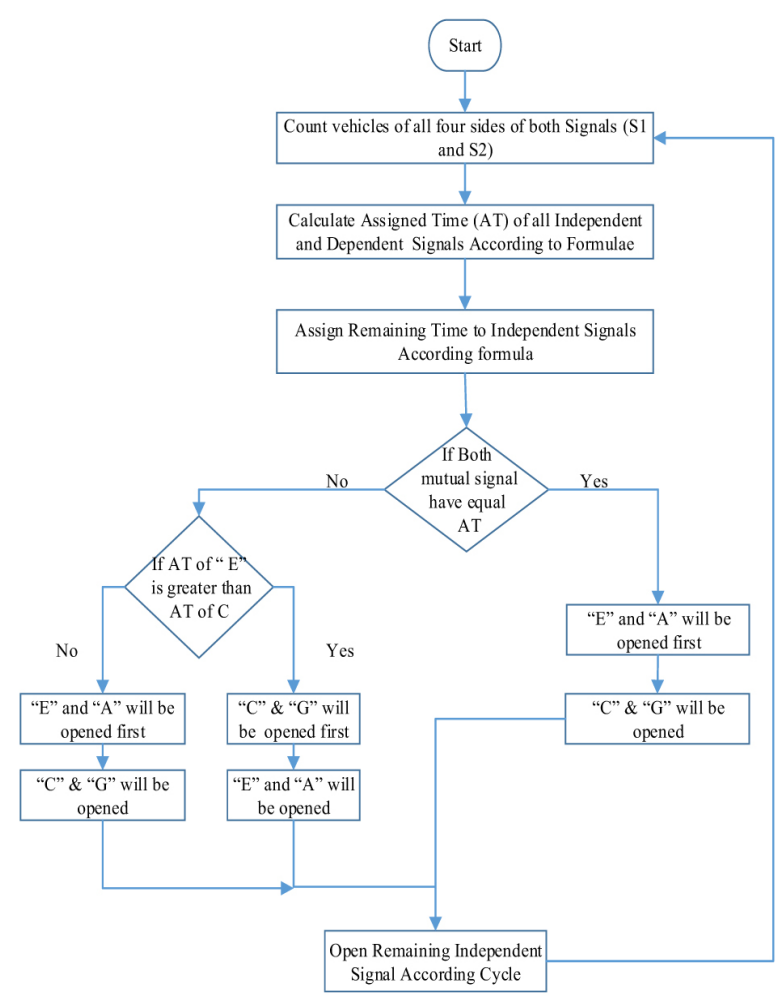

Fig. 6. Mutual Signal Assign Time Algorithm

\section{CASE STUDY}

Traffic is the common problem in large modern cities as well as in third world countries. Developed countries have already paid keen attention to the traffic congestion problem and are solving it by adopting intelligent strategies. Traffic signals are divided into two types of signals i.e. independent and mutual dependent signals. The solution of mutual dependent signal was discussed in this section, whereas the independent signals have already been discussed in our previous paper [30].

Two signals which are connected and close to each other are said to be mutual dependent traffic signals. In the case of mutual dependent traffic signals, the traffic flow of one signal affects the traffic flow of the other signal and vice versa. There are 7 roads and each road has two sub-roads i.e. incoming and outgoing, as shown in Figure 7. The assigned time of the signal ' $\mathrm{C}$ ' depends on the vehicles that are waiting on Signal ' $C$ ' and outgoing traffic of signal ' $G$ '. The case for signal ' $\mathrm{E}$ ' is similar; the assigned time depends on the vehicles waiting on ' $\mathrm{A}$ '.

Let us consider a mutual dependent traffic signal where the number of vehicles at signal A, $\mathrm{B}, \mathrm{C}$ and $\mathrm{D}$ are 13, 20, 10 and 11 while at signal
E, F, G and $\mathrm{H}$ are 8, 21, 27 and 13 respectively. The above-mentioned mutual-dependent traffic signals can be represented through Figure 9, where each signal is denoted through a node and the incoming edge represents traffic or number of vehicles moving towards that node. The signals of Figure 7 are divided into two types, as shown in Figure 8, i.e. independent (A, B, D, F, G and H) - only depending on their own traffic, dependent (E, C) depends on its own and the vehicles waiting at signal $(A, G)$ respectively.

The above-mentioned automata representation can easily be understandable through an adjacency list. In graph theory, an adjacency list is a way to represent the nodes with their connectivity to other nodes. The first row of Table 1 represents the individual signal of the scenario above, while the second row shows the dependent signals.

As the signal A, B, D, F, G and $\mathrm{H}$ are independent signals; that is why they were calculated according to the independent signal scenario. However, the assigned time and remaining assigned time (RTA) of the mutual dependent signals were calculated by using following formulae:

Assigned Time of $C=$ No of vehicles $(C+0.7 G)(1)$

Assigned Time of $E=$ No of vehicles $(E+0.7 A)$ (2)

$R T A$ of Signal $B=(V B / V B+V D) \cdot R T$

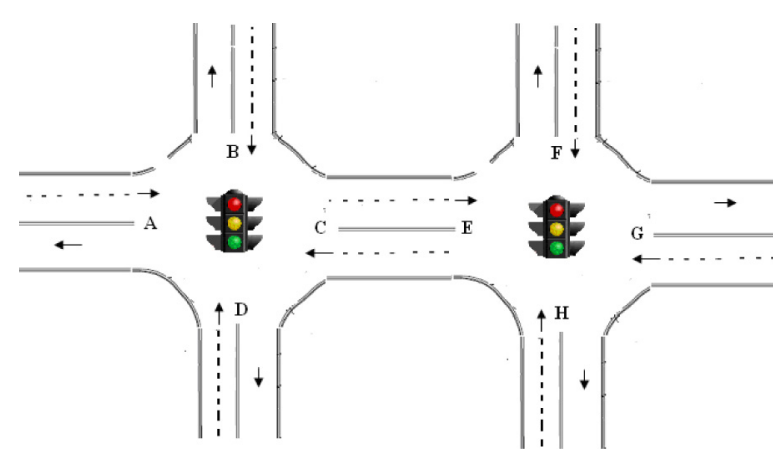

Fig. 7. Mutual-Dependent Traffic Signal Scenario

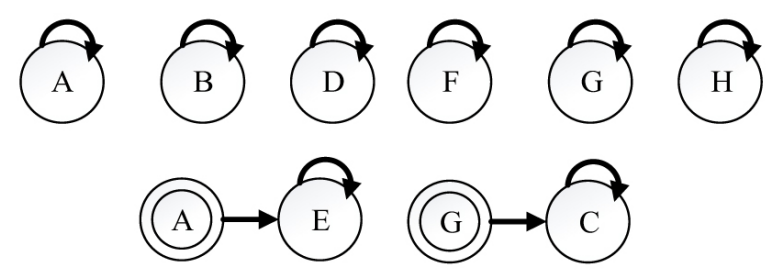

Fig. 8. Graph representation of Mutual-Dependent Traffic Signal 
Table 1. Signals with Associated Dependency

\begin{tabular}{|l|l|l|l|l|l|l|l|l|}
\hline $\begin{array}{l}\text { Independent } \\
\text { Signals }\end{array}$ & A & B & & D & & F & G & H \\
\hline $\begin{array}{l}\text { Dependent } \\
\text { Signals }\end{array}$ & & & C & & E & & & \\
\hline
\end{tabular}

In the equations above (1 and 2), 0.7 represents $70 \%$ of the total vehicles that approached towards the respective signal. These ratios were taken very carefully and with various traffic flow scenarios. After experimenting, it was found that $70 \%$ of the traffic moved towards dependent signal if it approached from the straight side. When the total time of both signals S1 and S2 are not same then RTA is added to the independent side of the signal having less time by using Eq (3), where calculation for only one signal is provided. In the same way, this formula (eq. 3) was adopted according to the specific side of each independent signal. Table 2 represents the assigned time and RTA to each signal by using the above-mentioned formulae.

\section{SIMULATION AND RESULTS}

Simulation provides the most effective way to handle and portray the real world problems. The traffic simulation can be categorized into deterministic (predictable) and stochastic [23, 27]. The deterministic simulation is the one which produces the same result for specific input; on the other hand, the stochastic simulation may produce dif- ferent outcome for same input. The other types of simulation are discrete or continuous. In discrete simulation, the system's states are modeled and their states vary after a specific interval or event (discrete time and discrete event simulation), while in the continuous simulation state of the model is determined by calculating the equations which are based on real values.

\section{Traffic Simulation Entitie and Environment}

Traffic simulation is used to simulate the flow of traffic in two types of vehicle traffic network, i.e. motorway and city traffic. However, here simulation was provided only for the city traffic. The main entities of the traffic include the cars, buses, roads and traffic signals. The traffic signals in this simulation were divided into independent and dependent traffic signal. There are four roads in the case of the independent traffic signal, while the dependent signal consists of two signals with six connecting roads. Vehicles and emergency vehicles are created on the roads randomly.

\section{Independent Traffic Signal}

The parameters used in calculation include the lanes, vehicles and priority in the case of emergency vehicles. The scenarios for independent traffic signals have already been discussed in [24] which showed that the ABAC architecture is more efficient than all other traffic control architectures.

Table 2. Results of Mutual Dependent Traffic Signals

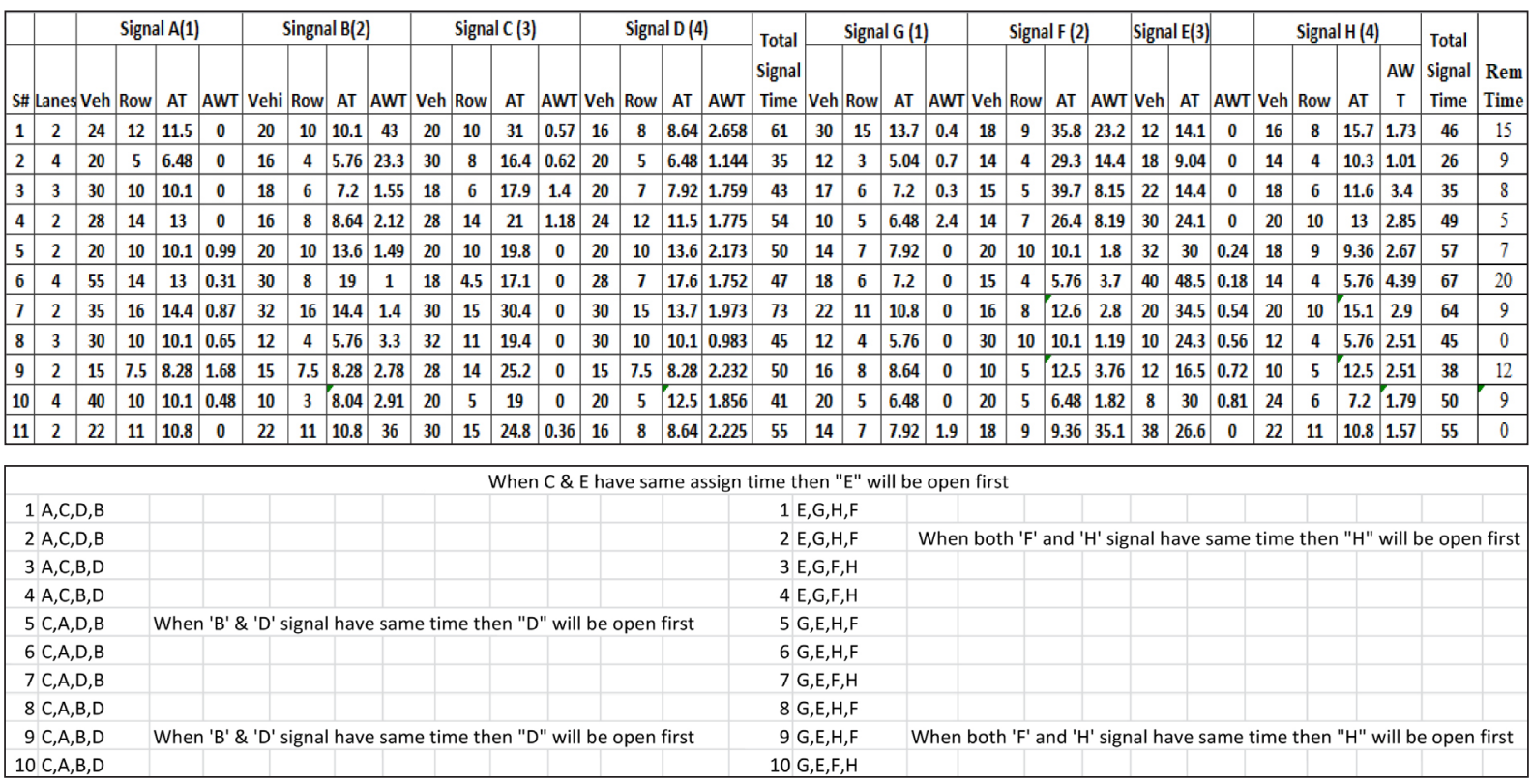




\section{Mutual-Dependent Traffic Signals}

In this section, more complex form of the traffic signals i.e. dependent signals was discussed. Two signals which are connected and close to each other are called dependent traffic signals. In the case of the dependent traffic signals, the traffic flow of one signal affects the other signal and vice versa, as shown in Figure 9.

In the scenario above, signals are opened according to their number of vehicles waiting at some signal as well as their dependent signal's traffic. The opening sequence of the dependent signal works according to vehicles.

\section{Mutual Dependent Traffic Signals with Rescue Vehicle}

Here, the case where the rescue vehicle appears on the interconnected signals was considered as shown in Figure 10. Priority of that road increases from where an emergency vehicle is coming and information about the emergency

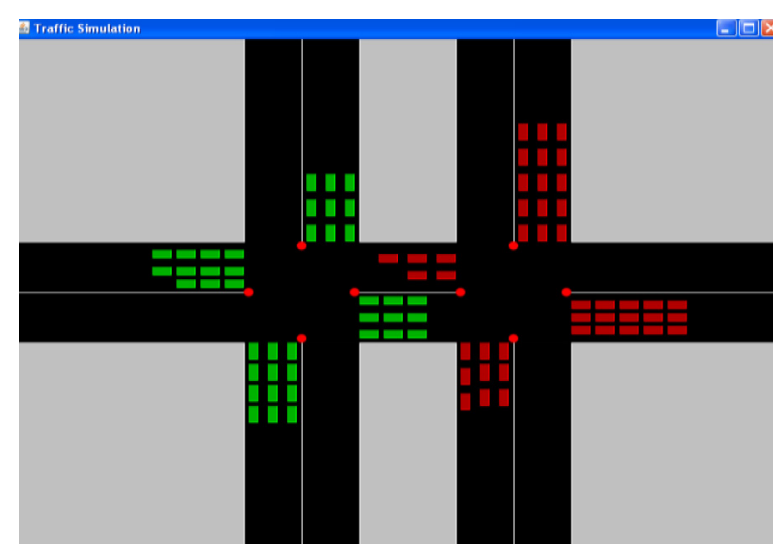

Fig. 9. Dependent Traffic Signal Scenario

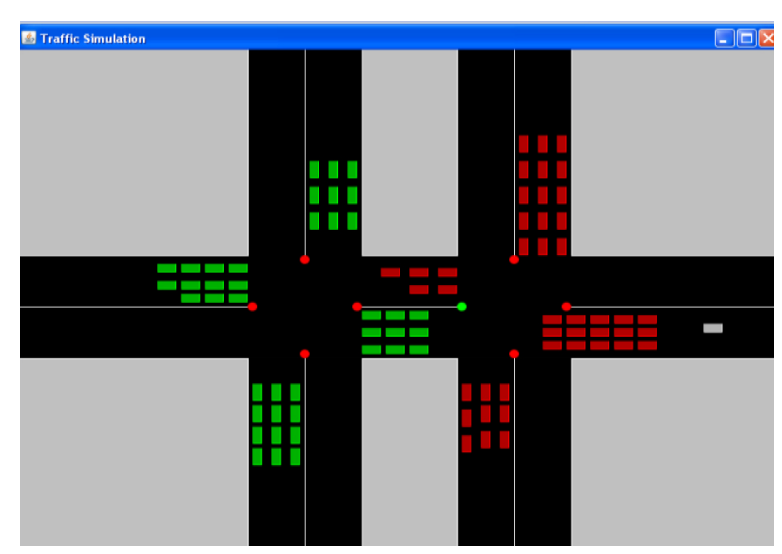

Fig. 10. Dependent Traffic Signal with emergency vehicle scenario vehicle is sent to the Decision Making agent of the next coming traffic signal. After obtaining this information, the next signal will modify its signal opening sequence in such a way so that the emergency vehicle passes away without any interference and delay.

In the above-mentioned scenario, an emergency vehicle is approaching to signal. Due to the emergency, the priority of this signal increases and signal is opened with $20 \mathrm{sec}$ more than the actual allocated time. After the passage of the emergency vehicle, DM agent is responsible for sending information to the next signal. After receiving this information, DM agent of next signal arranges the smooth passage of the emergency vehicle on first priority and after the emergency vehicle passage, the DM will resume signal to their previous sequence.

\section{CONCLUSIONS}

An autonomous agent-oriented traffic control system which manages the traffic flow on signals was proposed to save time. It manages the traffic flow without any human intervention. Vehicles are counted by the IR sensor. The algorithm was purposed to allocate time for mutual dependent signals. The emergency vehicles were given priority over public vehicles by using RFID tags. The research handles the traffic congestion problem intelligently and validated it through a simulation. The purposed system was demonstrated through simulation by taking different traffic scenarios. In future, this architecture can be integrated with other systems such as traffic bulletin boards that show traffic congestion on next signal or road.

\section{Acknowledgements}

We are grateful to the Higher Education Commission (HEC) of Pakistan who are supporting this research work.

\section{REFERENCES}

1. Mueller E.A., Aspects of the history of traffic signals. IEEE T. on Vehicular Technology, 19(1), 1970, 6-17.

2. Horn P., Autonomic Computing: IBM's Perspective on the State of Information Technology, IBM Journal, 2001. 
3. Kephart J.O. and Chess D.M., The Vision of Autonomic Computing. Computer, 36(1), 2003, 41-50.

4. An Architectural blueprint for autonomic computing, IBM White paper, $3^{\text {rd }}$ Edition, 2005.

5. Huebscher M.C.J. and Mccann A., A survey of Autonomic Computing Degrees, Models, \& Applications", ACM Computing Surveys, 40(3), 2008.

6. Stuart R. and Peter N., Artificial Intelligence, A Modern Approach, 2 ${ }^{\text {nd }}$ Edition, 81-7758-367-0, 2008.

7. Alagar V.S. and Muthiayen D., A Rigorous Approach to Modeling Autonomous Traffic Control Systems. In: The $6^{\text {th }}$ International Symposium on Autonomous Decentralized Sys., Italy, 2003, 193-200.

8. Shamshirband S.S., Shirgahi H., Gholami M. and Kia B., Coordination between Traffic Signals Based on Cooperative, World App. Sc. J. Vol. 5 (5), 2008, 525-530.

9. Liu X. and Fang Z., An Agent-Based Intelligent Transport System. In: $11^{\text {th }}$ Int. Conference on Computer Supported Cooperative Work in Design, 2007, 304-315.

10. Casey M., MPEG-7 Sound Recognition Tools, Mitsubishi Electric Research Labs, Cambridge, MA, USA.

11. Albagul A., Hrairi M., Wahyudi, and Hidayathullah M.F., Design \& Development of Sensor Based Traffic Light System, American J. of App. Sc. 3 (3), 2006, 1745-1749.

12. Huang Y., Design of Traffic Light Control Systems Using Statecharts, The Computer Journal, 49(6), 2006.

13. Rajeshwari S., Hebbar S. and Golla V., Implementing Intelligent Traffic Control System for Congestion Control, Ambulance Clearance and Stolen Vehicle Detection.

14. Bode M., Jha S.S. and Nair S.B. A mobile agent based autonomous partial green corridor discovery and maintenance mechanism for emergency services amidst urban traffic. In: Proceedings of $1^{\text {st }}$ Int. Conference on IoT in Urban Space, 2014, pp. 13-18.

15. Bharadwaj R., Deepak J., Baranitharan M. and Vaidehi V.V. Efficient dynamic traffic control system using wireless sensor networks. In: Recent Trends in Information Technology (ICRTIT), International Conference on, IEEE, 2013, 668-673.

16. Nafi N.S., Khan R.H., Khan J.Y. and Gregory M. A predictive road traffic management system based on vehicular ad-hoc network. In: Telecommunication Networks and Applications Conference, Australasian, IEEE, 2014, 135-140.
17. Grover S., Saxena V.S. and Vatwani T. Design of intelligent traffic control system using image segmentation. Int. J. of Advances in Eng. \& Tech., 7(5), 2014.

18. El-Tantawy S., Abdulhai B. and Abdelgawad H. Multiagent reinforcement learning for integrated network of adaptive traffic signal controllers (MARLIN-ATSC): Methodology and large-scale application on downtown Toronto. Intelligent Transportation Systems. IEEE Transactions on, 14(3), 2013, 1140-1150.

19. 1lmudin A., Hashim M.H.A., Ja'afar N.M.Z., Salleh A.S., Jaafar A., and Sam M.F.M. Traffic Light Control System using $434 \mathrm{MHz}$ Radio Frequency. Int. J. of Research in Advent Technology, 2(8), 2014, 26-31.

20. Latha J.R. and Suman U. Intelligent Traffic Light Controller. International Journal, 38, 2015.

21. Ramzanzad M. and Kanan H.R. A new method for design and implementation of intelligent traffic control system based on fuzzy logic using FPGA. In IEEE Fuzzy Systems, $13^{\text {th }}$ Iranian Conference, 2013, 1-4.

22. Alam M., Chowdhury M., and Purohit P. Development of an Intelligent Traffic Management System Based on Modified Round-Robin Algorithm Emergency, 7(12), 2014.

23. Champion A., Mandiau R., Kolski C., Heidet A. and Kemeny A. Traffic Generation with the SCANeR II Simulator: Towards Mulit-Agent Architecture. In: Proceedings of the first Driving Simulation Conference, 1999, 311-324.

24. Elementz Engineers Private Limited, July 2018 http://www.elementzonline.com/ir-infrared-obstacle-avoidance-sensor-module

25. Mreeco Electronics, July 2018, http://www.mreeco.com/product/ir-infrared-obstacle-avoidancesensor-module

26. Electronic Hub, August 2018, http://www.electronicshub.org/ir-sensor/

27. Traffic Simulation with Matlab, July 2018, https:// www.elprocus.com/rfid-basic-introduction-simple-application

28. Arduino, September 2018. https://create.arduino. $\mathrm{cc} /$ projecthub/gadget-programmers/online-attendance-system-without-ethernet-c07682

29. Elecrow Technology, September 2018, https:// www.elecrow.com/download/HC-12.pdf

30. Mateen A., Khalid A., Khan L., Majeed S., and Akhtar T. Vigorous algorithms to control urban vehicle traffic. ICIS 2016:1-5. 